\title{
Set-theoretic constructions of two-point sets
}

\author{
by \\ Ben Chad, Robin Knight and Rolf Suabedissen (Oxford)
}

\begin{abstract}
A two-point set is a subset of the plane which meets every line in exactly two points. By working in models of set theory other than ZFC, we demonstrate two new constructions of two-point sets. Our first construction shows that in $\mathrm{ZFC}+\mathrm{CH}$ there exist two-point sets which are contained within the union of a countable collection of concentric circles. Our second construction shows that in certain models of ZF, we can show the existence of two-point sets without explicitly invoking the Axiom of Choice.
\end{abstract}

1. Introduction. Given a cardinal $\kappa$, a subset of the plane is said to be a $\kappa$-point set if and only if it meets every line in exactly $\kappa$ many points and is said to be a partial $\kappa$-point set if and only if it meets every line in at most $\kappa$ many points. We are particularly interested in the case that $\kappa=2$, and we refer to such sets as two-point sets.

The existence of two-point sets was first shown by Mazurkiewicz [8]. (A French translation is given in [9].) A two-point set is classically constructed via transfinite recursion, making use of an arbitrary well-ordering of the collection of all lines in the plane. We will demonstrate two constructions of two-point sets which result by varying the use of this well-ordering.

The first construction shows that working in $\mathrm{ZFC}+\mathrm{CH}$, we can construct a two-point set which is contained in the union of a countable collection of concentric circles of unbounded radius. In general, classical constructions of two-point sets can be thought of as starting with a c-point set and then refining it to a two-point set, and so this construction is of interest because we obtain a two-point set by refining an $\aleph_{0}$-point set. We achieve this development by being careful about the well-ordering of the collection of all lines that we choose to help us in our construction.

The second construction shows that in ZF, if we assume that the real line satisfies a certain number of properties, we can construct a two-point set without explicitly invoking the Axiom of Choice. It is a question of Erdős,

Key words and phrases: two-point set, continuum hypothesis, axiom of choice. 
as discussed by Mauldin [7], to determine if a two-point set can be a Borel set. It is known from work of Larman [6] and Baston and Bostock [1] that a two-point set cannot be an $F_{\sigma}$ set, but we know little else about this problem. Since a Borel set can always be described by a countable Borel code and the known methods of constructing two-point sets use a transfinite recursion of length $\mathfrak{c}$, it would appear that these existing techniques cannot possibly yield a two-point set which is guaranteed to be Borel. Accordingly, new methods of constructing two-point sets are necessary, which do not rely so explicitly on the Axiom of Choice. In our construction, the assumptions that we will make about the real line are implied by, and appear to be strictly weaker than, the assumption that it is well-orderable. If it indeed turns out to be the case that our assumptions are equivalent in $\mathrm{ZF}$ to the real line being well-orderable, our construction is still of interest, since we construct a two-point set by "dealing with" collections of lines simultaneously, rather than by "dealing with" lines sequentially.

The proof of the following result is essentially that given by Mazurkiewicz, and we include it for comparison.

TheOREm 1.1. There exists a two-point set.

Proof. Note that we can always extend a given partial two-point set with cardinality less than $\mathfrak{c}$ to a partial two-point set which meets a given line in exactly two points. To construct a two-point set we enumerate the set of all lines in order type $\mathfrak{c}$, and construct an increasing $\mathfrak{c}$-sequence of partial two-point sets, using the enumeration to ensure that the union of the members of the sequence is a two-point set.

Throughout the remainder of this paper, we use the variables $\alpha, \beta, \delta$ and $\gamma$ to range over ordinals, and the variables $\kappa$ and $\lambda$ to range over (wellorderable) cardinals.

2. The existence of two-point sets contained in $\aleph_{0}$-point sets. Given a property $P$, the proofs of the existence of $P$ two-point sets previously found in mathematical literature classically proceed in the manner of Theorem 1.1, and consist of a recursion of length $\mathfrak{c}$ which at each step requires a choice to be made from $\mathfrak{c}$ many possibilities. It follows that theorems of the form "There exists a two-point set with $P$ " which are proved in this way can be stated as theorems of the form "There exist $2^{\mathfrak{c}}$ two-point sets with $P$ ". Hence, if there exists some property $P$ (expressible in the language of set theory) such that the collection of $P$ two-point sets has cardinality less than $2^{\mathfrak{c}}$, then we cannot demonstrate that this is the case by constructing a $P$ two-point set with our classical techniques.

Isometry groups of two-point sets have been studied by Chad and Suabedissen. In [3], it was shown that any subgroup of $S^{1}$ of cardinality less than 
$\mathfrak{c}$ is the isometry group of some two-point set and that there exist proper subgroups of $S^{1}$ of cardinality c which are the isometry group of some two-point set. In [2], it was shown that there exist proper subgroups of $S^{1}$ which are not the isometry group of any two-point set. Given $G \leq S^{1}$ with coset representatives enumerated by $\left\langle g_{\alpha}: \alpha<\kappa\right\rangle$, a two-point set $X$ with isometry group $G$ is of the form $\bigcup_{\alpha<\kappa}\left(r_{\alpha} g_{\alpha} G \cup s_{\alpha} g_{\alpha} G\right)$ for some real-valued sequences $\left\langle r_{\alpha}: \alpha<\kappa\right\rangle$ and $\left\langle s_{\alpha}: \alpha<\kappa\right\rangle$. (To see this, note that $X$ is a union of $G$-orbits, and the line spanned by the origin and $g_{\alpha}$ meets $X$ in exactly two points.) Hence, if $G$ is chosen to have countable index, then there are at most $\mathfrak{c}$ many two-point sets with isometry group $G$. Further, if $X$ is indeed a two-point set with such an isometry group, then $X$ is contained in a union of countably many circles. While it was tempting for the authors to conjecture that it is impossible for a two-point set to be contained in a union of countably many circles, we will now demonstrate that this may possibly be the case.

We now introduce some terminology. Let $Y$ be an $\aleph_{0}$-point set and let $a, b, c \in Y$ be distinct. Then we say that $x \in Y \backslash\{a, b, c\}$ is a focus point with respect to $a, b$ and $c$ if and only if there is a line $L$ and $u, v, w \in L \cap Y$ such that $a, x$ and $u$ are collinear; as are $b, x$ and $v$; as are $c, x$ and $w$. Any line $L$ witnessing that $x$ is a focus point is called a screen. We note that if $Y$ has at most countably many focus points, then $Y$ has at most countably many screens.

For $A \subseteq \mathbb{R}^{2}$, we let $\mathcal{L}(A)$ denote the set of all lines spanned by distinct points from $A$. Note that $|\mathcal{L}(A)| \leq|A|+\aleph_{0}$. Further, for $B \subseteq \mathbb{R}^{2}$, we let $\mathcal{L}(A, B)$ denote the set of all lines spanned by a point from $A$ and a (different) point from $B$. It is easily seen that if $B \subseteq A$ then

$$
\mathcal{L}(A)=\mathcal{L}(B) \cup \mathcal{L}(B, A \backslash B) \cup \mathcal{L}(A \backslash B) .
$$

Again, let $Y$ be an $\aleph_{0}$-point set and let $A \subseteq \mathbb{R}^{2}$ be infinite. We say that $A$ is fat with respect to $Y$ if and only if for all $L \in \mathcal{L}(A)$ :

(1) $L \cap Y \subseteq A$;

(2) $\{x \in Y: x$ is a focus point with respect to a triple in $A \cap Y\} \subseteq A$;

(3) $\bigcup\{L \cap Y: L$ is a screen with respect to a triple in $A \cap Y\} \subseteq A$.

LEMma 2.1. Let $Y$ be an $\aleph_{0}$-point set such that each triple of distinct points in $Y$ has at most countably many focus points, and let $A \subseteq \mathbb{R}^{2}$ be countably infinite. Then there exists $B \subseteq \mathbb{R}^{2}$ such that $A \subseteq B, B$ is countably infinite and $B$ is fat with respect to $Y$. Further, $B$ may be chosen to be minimal with respect to set inclusion.

Proof. Let $T_{1}, T_{2}$ and $T_{3}$ be transformations of $\mathcal{P}\left(\mathbb{R}^{2}\right)$ such that for all $X \subseteq \mathbb{R}^{2}$,

$$
T_{1}(X)=\bigcup\{L \cap Y: L \in \mathcal{L}(X)\}
$$


$T_{2}(X)=\{x \in Y: x$ is a focus point with respect to a triple in $X \cap Y\}$, $T_{3}(X)=\bigcup\{L \cap Y: L$ is a screen with respect to a triple in $X \cap Y\}$.

Let $\left\langle B_{n}: n<\omega\right\rangle$ be an increasing sequence defined for $n<\omega$ by $B_{0}=A$ and

$$
B_{n+1}=B_{n} \cup T_{1}\left(B_{n}\right) \cup T_{2}\left(B_{n}\right) \cup T_{3}\left(B_{n}\right),
$$

and let $B=\bigcup_{n<\omega} B_{n}$. Then it is easily verified that $B$ is countably infinite, that $B$ is fat with respect to $Y$ and that $B$ is minimal.

We now introduce our final pieces of terminology. For each $A \subseteq \mathbb{R}^{2}$ and each partial two-point set $X \subseteq A$, we say that $X$ is full with respect to $A$ if and only if $\mathcal{L}(X)=\mathcal{L}(A)$. We say that $Z \subseteq \mathbb{R}^{2}$ is a finite extension of a partial two-point set $X$ if and only if $Z$ is a partial two-point set such that $Z \supseteq X$ and $|Z \backslash X|<\aleph_{0}$. Given an $\aleph_{0}$-point set $Y$ and a partial two-point set $X \subseteq Y$, we let $\mathbb{P}(X, Y)$ be the set $\{Z \subseteq Y: Z$ is a finite extension of $X\}$ equipped with its partial order of reverse inclusion.

Recall that given a partial order $\langle\mathbb{P}, \leq\rangle$, a subset $D$ of $\mathbb{P}$ is dense in $\mathbb{P}$ if and only if for every $p \in \mathbb{P}$ there is $d \in D$ such that $d \leq p$.

Lemma 2.2. Let $Y$ be an $\aleph_{0}$-point set, let $A \subseteq \mathbb{R}^{2}$ be fat with respect to $Y$ and let $X \subseteq A$ be a partial two-point set such that $X$ is full with respect to $A$. Then for all lines $L,\{Z \in \mathbb{P}(X, Y):|L \cap Z|=2\}$ is dense in $\mathbb{P}(X, Y)$.

Proof. Let $L$ be a line and let $W \in \mathbb{P}(X, Y)$ be such that $|L \cap W|<2$. Then $L \notin \mathcal{L}(A)$, for otherwise $L \in \mathcal{L}(X) \subseteq \mathcal{L}(W)$. Suppose that there does not exist a finite extension $Z \subseteq Y$ of $W$ such that $L \in \mathcal{L}(Z)$. Then every point of $L \cap Y$, except perhaps one, belongs to a member of $\mathcal{L}(W)$. We will show that each of

$$
L \cap Y \cap \bigcup \mathcal{L}(X), \quad L \cap Y \cap \bigcup \mathcal{L}(X, W \backslash X), \quad L \cap Y \cap \bigcup \mathcal{L}(W \backslash X)
$$

is finite, which is then clearly a contradiction.

Suppose that there exist distinct $x_{1}, x_{2} \in L \cap Y \cap(\bigcup \mathcal{L}(X))$. Then $x_{1} \in L_{1}$ and $x_{2} \in L_{2}$ for some $L_{1}, L_{2} \in \mathcal{L}(X)=\mathcal{L}(A)$, and so by fatness, both $x_{1}$ and $x_{2}$ belong to $A$, whence by fullness we obtain the contradiction that $L \in \mathcal{L}(X)$.

Now, $\mathcal{L}(X, W \backslash X)=\bigcup_{w \in W \backslash X} \mathcal{L}(X,\{w\})$, and it is easily seen that each $L \cap Y \cap \mathcal{L}(X,\{w\})$ is at most a pair set, otherwise $L$ is a screen with respect to a triple in $X \subseteq A \cap Y$. Since $W \backslash X$ is finite, it follows that $L \cap Y \cap \bigcup \mathcal{L}(W \backslash X)$ is finite.

Since $\mathcal{L}(W \backslash X)$ is finite, it is obvious that $L \cap Y \cap \bigcup \mathcal{L}(W \backslash X)$ is finite.

We are now in a position to prove the first of our two main results. Our proof is similar to that of Theorem 1.1, which treats lines sequentially, the main difference being that we do not trust an arbitrary well-ordering of the 
collection of all lines to be sufficient for our purposes. Intuitively, extending a countable partial two-point set $X$ to meet some countable collection $\mathcal{K}$ of lines in exactly two points may create a situation where there exist lines which our new partial two-point set cannot be extended to (within our given $\aleph_{0}$-point set). The preceding lemmas allow us to identify the family $\mathcal{K}^{\prime}$ of lines which may be in future danger of witnessing this problem, and since $\mathcal{K}^{\prime}$ is also countable, instead of extending $X$ to meet all members of $\mathcal{K}$ in exactly two points, we extend it to meet all members of $\mathcal{K} \cup \mathcal{K}^{\prime}$ in exactly two points.

THeOREM $2.3(\mathrm{ZFC}+\mathrm{CH})$. Let $Y$ be an $\aleph_{0}$-point set such that each triple of distinct points in $Y$ has at most countably many focus points. Then $Y$ contains a two-point set.

Proof. By applying Lemma 2.1, there exists a sequence $\left\langle A_{\alpha}: \alpha<\mathfrak{c}\right\rangle$ of increasing countable sets which are fat with respect to $Y$ and such that $\bigcup_{\alpha<\mathfrak{c}} A_{\alpha}=\mathbb{R}^{2}$. We will construct an increasing sequence $\left\{X_{\alpha}: \alpha<\mathfrak{c}\right\}$ of partial two-point sets such that each $\bigcup_{\beta \leq \alpha} X_{\beta}$ is a countable set which is full with respect to $A_{\alpha}$ and $\bigcup_{\alpha<\mathfrak{c}} X_{\alpha}$ is a two-point set. Suppose that the partial sequence $\left\langle X_{\beta}: \beta<\alpha\right\rangle$ has been chosen. Then $\mathcal{L}\left(A_{\alpha}\right)$ is countable, and so by Lemma 2.2 and the Rasiowa-Sikorski Lemma [5], there exists a filter $\mathcal{F}$ in $\mathbb{P}\left(\bigcup_{\beta<\alpha} X_{\beta}, Y\right)$ such that for all $L \in \mathcal{L}\left(A_{\alpha}\right)$ there exists $F \in \mathcal{F}$ such that $L \in \mathcal{L}(F)$. Hence, letting $X_{\alpha}=(\bigcup \mathcal{F}) \cap A_{\alpha}$, we have a countable partial two-point set which extends $\bigcup_{\beta<\alpha} X_{\beta}$ and is full with respect to $A_{\alpha}$.

Now letting the $X_{\alpha}$ be chosen for all $\alpha<\mathfrak{c}$, we see that $\bigcup_{\alpha<\mathfrak{c}} X_{\alpha}$ is a two-point set contained in $Y$.

\section{The existence of two-point sets in a union of countably many} concentric circles. We now show that a union of countably many concentric circles of unbounded radii consistently contains two-point sets. Let $S^{1}$ denote the unit circle in $\mathbb{C}$, and for all $z_{\alpha}, z_{\beta}, z_{\gamma} \in \mathbb{C}$ and all $r_{\alpha}, r_{\beta}, r_{\gamma} \in \mathbb{R}^{+}$, let $\mathrm{E}\left(z_{\alpha}, z_{\beta}, z_{\gamma}, r_{\alpha}, r_{\beta}, r_{\gamma}\right)$ denote the (formal) expression

$$
r_{\alpha} r_{\beta} z_{\gamma}\left(z_{\alpha}^{2}-z_{\beta}^{2}\right)+r_{\beta} r_{\gamma} z_{\alpha}\left(z_{\beta}^{2}-z_{\gamma}^{2}\right)+r_{\gamma} r_{\alpha} z_{\beta}\left(z_{\gamma}^{2}-z_{\alpha}^{2}\right),
$$

which is derived from writing down the length of the vector product

$$
\left(z_{\alpha}-z_{\gamma}\right) \times\left(z_{\beta}-z_{\gamma}\right),
$$

where the complex numbers are thought of as being vectors in the plane. Note that for all $z_{\alpha}, z_{\beta}, z_{\gamma} \in \mathbb{C} \backslash\{0\}$, it can be seen that $z_{\alpha}, z_{\beta}$ and $z_{\gamma}$ are collinear over $\mathbb{R}$ if and only if

$$
\mathrm{E}\left(\frac{z_{\alpha}}{\left|z_{\alpha}\right|}, \frac{z_{\beta}}{\left|z_{\beta}\right|}, \frac{z_{\gamma}}{\left|z_{\gamma}\right|},\left|z_{\alpha}\right|,\left|z_{\beta}\right|,\left|z_{\gamma}\right|\right)=0 .
$$


Further, for all $z_{\alpha}, z_{\beta}, z_{\gamma} \in \mathbb{C}$ and all $r_{\alpha}, r_{\beta}, r_{\gamma} \in \mathbb{R}^{+}$, it can be seen that

$$
\left\langle z_{\alpha}^{2}, z_{\alpha} / r_{\alpha}\right\rangle,\left\langle z_{\beta}^{2}, z_{\beta} / r_{\beta}\right\rangle,\left\langle z_{\gamma}^{2}, z_{\gamma} / r_{\gamma}\right\rangle \in \mathbb{C}^{2}
$$

are collinear over $\mathbb{C}$ if and only if $\mathrm{E}\left(z_{\alpha}, z_{\beta}, z_{\gamma}, r_{\alpha}, r_{\beta}, r_{\gamma}\right)=0$. For all $r_{a}, r_{b}, r_{c}$, $r_{x}, r_{u}, r_{v}, r_{w} \in \mathbb{R}^{+}$and all $z_{a}, z_{b}, z_{c}, z_{x}, z_{u}, z_{v}, z_{w} \in \mathbb{C}$, let

$$
\phi\left(z_{a}, z_{b}, z_{c}, z_{x}, z_{u}, z_{v}, z_{w}, r_{a}, r_{b}, r_{c}, r_{x}, r_{u}, r_{v}, r_{w}\right)
$$

be a sentence which is true if and only if each of $\mathrm{E}\left(z_{a}, z_{x}, z_{u}, r_{a}, r_{x}, r_{u}\right)$, $\mathrm{E}\left(z_{b}, z_{x}, z_{v}, r_{b}, r_{x}, r_{v}\right), \mathrm{E}\left(z_{c}, z_{x}, z_{w}, r_{c}, r_{z}, r_{w}\right)$ and $\mathrm{E}\left(z_{u}, z_{v}, z_{w}, r_{u}, r_{v}, r_{w}\right)$ are equal to zero.

LEMMA 3.1. Let $r_{a}, r_{b}, r_{c}, r_{x}, r_{u}, r_{v}, r_{w} \in \mathbb{R}^{+}$, let $z_{a}, z_{b}, z_{c} \in S^{1}$ be such that $r_{a} z_{a}, r_{b} z_{b}, r_{c} z_{c} \in \mathbb{C}$ are distinct, let $S$ be the set $\left\{z_{x} \in S^{1}: \exists z_{u}, z_{v}, z_{w} \in S^{1} \phi\left(z_{a}, z_{b}, z_{c}, z_{x}, z_{u}, z_{v}, z_{w}, r_{a}, r_{b}, r_{c}, r_{x}, r_{u}, r_{v}, r_{w}\right)\right\}$, and let $T$ be the set

$$
\left\{z_{x} \in \mathbb{C}: \exists z_{u}, z_{v}, z_{w} \in \mathbb{C} \phi\left(z_{a}, z_{b}, z_{c}, z_{x}, z_{u}, z_{v}, z_{w}, r_{a}, r_{b}, r_{c}, r_{x}, r_{u}, r_{v}, r_{w}\right)\right\} .
$$

If $S$ is uncountable then $T=\mathbb{C}$.

Proof. For each $z_{x} \in \mathbb{C}$, we consider $\mathrm{E}\left(z_{a}, z_{x}, z_{u}, r_{a}, r_{x}, r_{u}\right)$ to be a polynomial in $z_{u}$, which is non-trivial when $z_{x} \neq r_{a} z_{a} / r_{x}$. By choosing one of finitely many branch cuts, each of $z_{u}, z_{v}, z_{w}$ can be viewed as a complex differentiable function of $z_{x}$. Supposing that $S$ is uncountable, let

$$
S^{\prime}=S \backslash\left\{\frac{r_{a} z_{a}}{r_{x}}, \frac{r_{b} z_{b}}{r_{x}}, \frac{r_{c} z_{c}}{r_{x}}\right\},
$$

which is uncountable. It can then be seen that some choice of branches takes the value zero uncountably often on $S^{\prime}$, and for this choice, we substitute $z_{u}$, $z_{v}, z_{w}$ as functions of $z_{x}$ into $\mathrm{E}\left(z_{u}, z_{v}, z_{w}, r_{u}, r_{v}, r_{w}\right)$. This gives us a complex differentiable function of $z_{x}$, except on perhaps the branch cuts. By choosing an appropriate branch cut and applying the Identity Theorem, the result then follows.

For each $r>0$, let $C_{r}$ denote the circle in $\mathbb{C}$ of radius $r$ and centred at the origin.

LEMMA 3.2. Let $a, b, c \in \mathbb{C} \backslash\{0\}$ be distinct, let $r_{x}, r_{u}, r_{v}, r_{w} \in \mathbb{R}^{+}$, and let $S$ be the set of all $x \in C_{r_{x}}$ such that there exist $u \in C_{r_{u}}, v \in C_{r_{v}}$ and $w \in C_{r_{w}}$ with the property that

$$
\phi\left(\frac{a}{|a|}, \frac{b}{|b|}, \frac{c}{|c|}, \frac{x}{|x|}, \frac{u}{|u|}, \frac{v}{|v|}, \frac{w}{|w|},|a|,|b|,|c|,|x|,|u|,|v|,|w|\right) .
$$

If $S$ is uncountable, then $a, b$ and $c$ are collinear.

Proof. Suppose that $S$ is uncountable. We write $r_{\alpha}=|\alpha|$ and $z_{\alpha}=\alpha / r_{\alpha}$ for $\alpha=a, b, c$. By elementary algebra, we can show that there exists $z_{x} \in \mathbb{C}$ 
such that

$$
\left\langle z_{a}^{2}, z_{a} / r_{a}\right\rangle,\left\langle z_{b}^{2}, z_{b} / r_{b}\right\rangle,\left\langle z_{x}^{2}, z_{x} / r_{x}\right\rangle \in \mathbb{C}^{2}
$$

are collinear over $\mathbb{C}$. Using Lemma 3.1, let $z_{u}, z_{v}, z_{w} \in \mathbb{C}$ be such that

$$
\begin{aligned}
\mathrm{E}\left(z_{a}, z_{x}, z_{u}, r_{a}, r_{x}, r_{u}\right) & =0, \\
\mathrm{E}\left(z_{b}, z_{x}, z_{v}, r_{b}, r_{x}, r_{v}\right) & =0, \\
\mathrm{E}\left(z_{c}, z_{x}, z_{w}, r_{c}, r_{x}, r_{w}\right) & =0, \\
\mathrm{E}\left(z_{u}, z_{v}, z_{w}, r_{u}, r_{v}, r_{w}\right) & =0 .
\end{aligned}
$$

Using (1), (2) and (3), we add $\left\langle z_{u}^{2}, z_{u} / r_{u}\right\rangle$ and $\left\langle z_{v}^{2}, z_{v} / r_{v}\right\rangle$ to the list of collinear points in (1), then using (4) we add $\left\langle z_{w}^{2}, z_{w} / r_{w}\right\rangle$ to the list, and finally using (3) we add $\left\langle z_{c}^{2}, z_{c} / r_{c}\right\rangle$ to the list. Thus $\mathrm{E}\left(z_{a}, z_{b}, z_{c}, r_{a}, r_{b}, r_{c}\right)=0$, giving that $a, b$ and $c$ are collinear over $\mathbb{R}$.

The following is now easily seen:

LEMMA 3.3. Let $\mathcal{C}$ be a collection of countably many concentric circles of unbounded radii and let $a, b, c \in \bigcup \mathcal{C}$ be distinct. Then there are only countably many focus points with respect to $a, b$ and $c$.

THEOREM $3.4(\mathrm{ZFC}+\mathrm{CH})$. Let $\mathcal{C}$ be a collection of countably many concentric circles of unbounded radii. Then $\bigcup \mathcal{C}$ contains a two-point set.

4. A non-sequential construction of a two-point set. Working in a certain model of $\mathrm{ZF}$, we will now construct a two-point set without explicitly invoking the Axiom of Choice. We achieve this by changing the strictly sequential nature of the classical construction, and instead of dealing with lines one-by-one, we deal with collections of lines simultaneously. We start with a technical lemma.

LEMMA 4.1. Let $L_{1}$ and $L_{2}$ be distinct lines and let $a \in \mathbb{R}^{2} \backslash\left(L_{1} \cup L_{2}\right)$. Then there are at most twenty-two $r>0$ such that there exist $b \in L_{1} \backslash L_{2}$ and $c \in L_{2} \backslash L_{1}$ having the properties that $a, b$ and $c$ are collinear and $\|b\|=\|c\|=r$.

Proof. For $r>0$, consider the circle $C=\left\{\langle x, y\rangle \in \mathbb{R}^{2}: x^{2}+y^{2}=r^{2}\right\}$. Given $x_{0} \in \mathbb{R}$ and a line of the form $L=\left\{\langle x, y\rangle \in \mathbb{R}^{2}: x=x_{0}\right\}$, the members of $C \cap L$ (if they exist) are of the form

$$
\left\langle x_{0}, \pm \sqrt{r^{2}-x_{0}^{2}}\right\rangle
$$

and given $m, b \in \mathbb{R}$ and a line of the form $L=\left\{\langle x, y\rangle \in \mathbb{R}^{2}: y=m x+b\right\}$, the members of $C \cap L$ (if they exist) are of the form

$$
\left\langle\frac{-m b \pm \sqrt{\left(m^{2}+1\right) r^{2}-b^{2}}}{m^{2}+1}, \frac{b \pm m \sqrt{\left(m^{2}+1\right) r^{2}-b^{2}}}{m^{2}+1}\right\rangle .
$$


Suppose that $r, b$ and $c$ are as in the statement of the lemma. By applying a rotation to our system, we may assume that $L_{1}$ is a vertical line with parameter $x_{1} \in \mathbb{R}$. We consider two cases, depending on the form of $L_{2}$.

CASE 1: $L_{2}=\left\{\langle x, y\rangle \in \mathbb{R}^{2}: x=x_{2}\right\}$ for some $x_{2} \in \mathbb{R}$. In this case,

$$
\frac{ \pm \sqrt{r^{2}-x_{1}^{2}}-a^{(2)}}{x_{1}-a^{(1)}}=\frac{ \pm \sqrt{r^{2}-x_{2}^{2}}-a^{(2)}}{x_{2}-a^{(1)}},
$$

and by repeated squaring to eliminate the radicals in this equality, we obtain a polynomial equation in $r$ whose highest formal power is 4 . If the coefficient of $r^{4}$ vanishes then the coefficient of $r^{2}$ does not vanish, and so the polynomial is non-trivial. Since the polynomial is of degree at most four, $r$ can be only one of four possibilities.

CASE 2: $L_{2}=\left\{\langle x, y\rangle \in \mathbb{R}^{2}: y=m x+b\right\}$ for some $m, b \in \mathbb{R}$. If the line spanned by $a, b$ and $c$ is vertical, then we can argue that

$$
\frac{r^{2}}{m^{2}+1}-\frac{b^{2}}{\left(m^{2}+1\right)^{2}}-\left(a^{(1)}+\frac{m b}{m^{2}+1}\right)^{2}=0 .
$$

This represents a non-trivial quadratic equation in $r$, and so $r$ can be only one of two possibilities. Otherwise, we have

$$
\frac{ \pm \sqrt{r^{2}-x_{1}^{2}}-a^{(2)}}{x_{1}-a^{(1)}}=\frac{b \pm m \sqrt{\left(m^{2}+1\right) r^{2}-b^{2}}-a^{(2)}\left(m^{2}+1\right)}{-m b \pm \sqrt{\left(m^{2}+1\right) r^{2}-b^{2}}-a^{(1)}\left(m^{2}+1\right)} .
$$

If we rationalise the denominator of the value on the right-hand side of this expression, make $\left(r^{2}-x_{1}^{2}\right) /\left(x_{1}-a^{(1)}\right)^{2}$ the subject, and multiply both sides of the resulting equality by

$$
\left(m^{2}+1\right) r^{2}-b^{2}-\left(-m b-a^{(1)}\left(m^{2}+1\right)\right)^{2},
$$

then the left-hand side is a rational function in $r$ with the coefficient of $r^{4}$ being $\left(m^{2}+1\right) /\left(x_{1}-a^{(1)}\right) \neq 0$, and the right-hand side is a rational function in $r$ with no occurrences of $r^{4}$. By repeated squaring to eliminate the radicals in this equality, we obtain a polynomial in $r$ of degree 16 with coefficient of $r^{16}$ being $\left(m^{2}+1\right) /\left(x_{1}-a^{(1)}\right) \neq 0$, and so $r$ can be only one of sixteen possibilities.

We are now in a position to demonstrate our construction. It is hoped the axioms required for this construction may be found to be true in some model of $\mathrm{ZF}+\neg \mathrm{AC}(\mathbb{R})$, but as we will later note, they are certainly true in some models of ZFC, and can be proved from $\mathrm{ZFC}+\mathrm{CH}$.

TheOREM $4.2(\mathrm{ZF})$. Suppose that there exists a well-orderable cardinal $\kappa$ such that:

(1) $\kappa^{+}$is regular; 
(2) $\mathbb{R}$ can be written as the union of a well-orderable and increasing collection of sets with cardinality $\kappa$;

(3) $\mathbb{R}$ contains a well-orderable set of cardinality $\kappa^{+}$.

Then there exists a two-point set.

Proof. By condition (2), there exists an increasing sequence $\left\langle A_{\alpha}: \alpha<\lambda\right\rangle$ of subsets of $\mathbb{R}$ where each $A_{\alpha}$ is of cardinality $\kappa$ and $\lambda$ is a well-orderable cardinal less than or equal to $\kappa^{+}$, and such that $\mathbb{R}=\bigcup_{\alpha<\lambda} A_{\alpha}$. By condition (3), there exists an injective sequence $\left\langle x_{\alpha}: \alpha<\kappa^{+}\right\rangle$on $\mathbb{R}$. By applying suitable transformations, we will assume that each $x_{\alpha}$ is positive, and that for all $M>0,\left\{\alpha<\kappa^{+}: x_{\alpha}>M\right\}$ is of cardinality $\kappa^{+}$.

Let $\left\langle\mathcal{L}_{\alpha}: \alpha<\lambda\right\rangle$ be an increasing sequence such that $\bigcup_{\alpha<\lambda} \mathcal{L}_{\alpha}$ is the collection of all lines in $\mathbb{R}^{2}$, where for all $\alpha<\lambda,\left|\mathcal{L}_{\alpha}\right|=\kappa$ and $\mathcal{L}_{\alpha+1} \backslash \mathcal{L}_{\alpha} \neq \emptyset$. We will construct our two-point set as an increasing union $\bigcup_{\alpha<\lambda} X_{\alpha}$, where for each $\alpha<\lambda, X_{\alpha}$ is a partial two-point set of cardinality $\kappa$ which meets every member of $\mathcal{L}_{\alpha}$ in exactly two points.

Suppose that for some $\alpha<\lambda$ we have constructed the partial sequence $\left\langle X_{\beta}: \beta<\alpha\right\rangle$. We now show how to extend $\bigcup_{\beta<\alpha} X_{\beta}$ to a partial two-point set $Y_{\alpha}$, where $Y_{\alpha} \backslash \bigcup_{\beta<\alpha} X_{\beta}$ contains exactly one point on each member of $\mathcal{L}_{\alpha}$ which does not meet $\bigcup_{\beta<\alpha} X_{\beta}$ in two points. Letting On denote the class of all ordinals, we will achieve this by constructing an increasing term $\left\langle X_{\alpha, \beta}: \beta \in \mathbf{O n}\right\rangle$ and a decreasing term $\left\langle\mathcal{L}_{\alpha, \beta}: \beta \in \mathbf{O n}\right\rangle$, both consisting of sets of cardinality at most $\kappa$. Let

$$
\begin{aligned}
& X_{\alpha, 0}=\bigcup_{\beta<\alpha} X_{\beta}, \\
& \mathcal{L}_{\alpha, 0}=\left\{L \in \mathcal{L}_{\alpha}: \bigcup_{\beta<\alpha} X_{\beta} \text { meets } L \text { in fewer than two points }\right\} .
\end{aligned}
$$

Suppose that for some $\beta \in \mathbf{O n} \backslash\{0\}$, we have chosen $\left\langle X_{\alpha, \gamma}: \gamma<\beta\right\rangle$ and $\left\langle\mathcal{L}_{\alpha, \gamma}: \gamma<\beta\right\rangle$. If $\bigcap_{\gamma<\beta} \mathcal{L}_{\alpha, \gamma}=\emptyset$ then we set $X_{\alpha, \beta}=\bigcup_{\gamma<\beta} X_{\alpha, \gamma}$ and $\mathcal{L}_{\alpha, \beta}=\emptyset$. Otherwise, let

$$
\mathcal{L}_{\alpha, \beta}=\left\{L \in \bigcap_{\gamma<\beta} \mathcal{L}_{\alpha, \gamma}:\left(\bigcup_{\gamma<\beta} X_{\alpha, \gamma} \backslash \bigcup_{\gamma<\alpha} X_{\gamma}\right) \cap L=\emptyset\right\} .
$$

Given a partial two-point set $P$, recall from Section 2 that $\mathcal{L}(P)$ denotes the set of lines meeting $P$ in exactly two points. Note that if $P$ contains at least two points, then $P \subseteq \bigcup \mathcal{L}(P)$. Let $\phi(L, \delta)$ be a sentence such that for $L \in \mathcal{L}_{\alpha, \beta}$ and $\delta<\kappa^{+}, \phi(L, \delta)$ is true if and only if:

there exists $b \in L \backslash \bigcup\left(\mathcal{L}\left(\bigcup_{\gamma<\beta} X_{\alpha, \gamma}\right) \cup \bigcup_{\gamma<\alpha} \mathcal{L}_{\gamma} \backslash\{L\}\right)$ such that $\|b\|=$ $x_{\delta}$ and for all $a$ and $c$ where $a \in \bigcup_{\gamma<\beta} X_{\alpha, \gamma}$ and $K \in \bigcap_{\gamma<\beta} \mathcal{L}_{\alpha, \gamma} \backslash\{L\}$ and $c \in K$ and $\|c\|=x_{\delta}$, the distinct points $a, b$ and $c$ are not collinear. 
Now, for all $L \in \mathcal{L}_{\alpha, \beta}$, the set $\left\{\delta<\kappa^{+}: \phi(L, \delta)\right\}$ is non-empty, since:

(1) $\mid\left\{\delta<\kappa^{+}: L\right.$ meets the circle of radius $\left.x_{\delta}\right\} \mid=\kappa^{+}$;

(2) $\bigcup_{\gamma<\beta} X_{\alpha, \gamma}$ and $\bigcap_{\gamma<\beta} \mathcal{L}_{\alpha, \gamma} \backslash\{L\}$ are of cardinality at most $\kappa$, and for all $a \in \bigcup_{\gamma<\beta} X_{\alpha, \gamma}$ and all $K \in \bigcap_{\gamma<\beta} \mathcal{L}_{\alpha, \gamma} \backslash\{L\}$, there are at most twenty-two $\delta$ such that we can find $b \in L$ and $c \in K$ with the properties that $a, b$ and $c$ are collinear and $\|b\|=\|c\|=x_{\delta}$;

(3) $\mathcal{L}\left(\bigcup_{\gamma<\beta} X_{\alpha, \gamma}\right) \cup \bigcup_{\gamma<\alpha} \mathcal{L}_{\gamma} \backslash\{L\}$ is of cardinality at most $\kappa$ and does not contain $L$.

(Note that in clause (2), we rely on the fact that from ZF we may prove the existence of a choice function on finite subsets of the plane.)

Let $f: \mathcal{L}_{\alpha, \beta} \rightarrow \kappa^{+}$be the function defined for $L \in \mathcal{L}_{\alpha, \beta}$ by

$$
f(L)=\min \left\{\delta<\kappa^{+}: \phi(L, \delta)\right\},
$$

let $\delta=\min \left\{f(L): L \in \mathcal{L}_{\alpha, \beta}\right\}$, and let $X_{\alpha, \beta}$ be chosen to enlarge $\bigcup_{\gamma<\beta} X_{\alpha, \gamma}$ by selecting one point on each $L$ such that $f(L)=\delta$. We can make such a selection without the aid of the Axiom of Choice, since we will only choose points with norm $x_{\delta}$, and we can deterministically choose between two points on a line of norm $x_{\delta}$. Clearly $X_{\alpha, \beta}$ is of cardinality at most $\kappa$, and we now verify that it is a partial two-point set. Let $L$ be any line. If $L$ does not meet $\bigcup_{\gamma<\beta} X_{\alpha, \gamma}$, then by selecting points in a (fixed) circle, we have ensured that $X_{\alpha, \beta}$ meets $L$ in at most two points, and if $L$ meets $\bigcup_{\gamma<\beta} X_{\alpha, \gamma}$ in exactly one (resp. two) point(s), then condition (2) (resp. (3)) above ensures that $X_{\alpha, \beta}$ meets $L$ in at most two points.

Consider the mapping $g: \kappa^{+} \rightarrow \mathcal{P}\left(\mathcal{L}_{\alpha}\right)$ defined for $\beta<\kappa^{+}$by

$$
g(\beta)=\mathcal{L}_{\alpha, \beta} \backslash \mathcal{L}_{\alpha, \beta+1} .
$$

Noting that $g$ is a disjointification of a decreasing sequence of subsets of $\mathcal{L}_{\alpha}$, it must eventually take the constant value $\emptyset$, otherwise $g$ is an injection from $\kappa^{+}$into a partition of $\bigcup_{\beta<\kappa^{+}} g(\beta) \subseteq \mathcal{L}_{\alpha}$, which is a contradiction. Hence, we set $Y_{\alpha}=\bigcup_{\beta<\alpha} X_{\alpha, \beta}$, which is an extension of $\bigcup_{\beta<\alpha} X_{\beta}$ meeting every member of $\mathcal{L}_{\alpha}$ in exactly one additional point. We now repeat the above process once more, extending $\bigcup_{\beta<\alpha} X_{\beta} \cup Y_{\alpha}$ instead of $\bigcup_{\beta<\alpha} X_{\beta}$ to obtain a partial two-point set $X_{\alpha}$ which meets every member of $\mathcal{L}_{\alpha}$ in exactly two points.

By working in ZFC and taking $\kappa=\aleph_{0}$, we see that:

TheOREM $4.3(\mathrm{ZFC}+\mathrm{CH})$. There exists a two-point set.

In comparison to the construction given in Sections 2 and 3, this construction also refines the union of some collection of concentric circles to a two-point set, and we have obtained its non-sequential nature at the price of making our collection of circles uncountable. 
This proof was originally developed with the hope that it could be made to work in a model of ZF where the reals can be written as a countable union of countable sets. The "standard model" of ZF where the reals have this property is due to Fefferman and Lévy and is described by Cohen [4]; in the Fefferman-Lévy model, the reals do not contain an uncountable well-ordered set. In response to private communication with the authors, Miller [10] has shown in that in any model of ZF where the reals are a countable union of countable sets, the reals do not contain an uncountable well-ordering, and also that by using the method of forcing, one can find models of ZF where the reals cannot be well-ordered, but which contain two-point sets.

\section{References}

[1] V. J. Baston and F. A. Bostock, On a theorem of Larman, J. London Math. Soc. (2) 5 (1972), 715-718.

[2] B. Chad and R. Suabedissen, On affine groups admitting invariant two-point sets, manuscript, http://people.maths.ox.ac.uk/chad, 2008.

[3] —, - Symmetries of two-point sets, Topology Appl. 155 (2008), 1213-1220.

[4] P. J. Cohen, Set Theory and the Continuum Hypothesis, Benjamin, New York, 1966.

[5] K. Kunen, Set Theory, Stud. Logic Found. Math. 102, North-Holland, Amsterdam, 1983.

[6] D. G. Larman, A problem of incidence, J. London Math. Soc. 43 (1968), 407-409.

[7] R. D. Mauldin, Problems in topology arising from analysis, in: Open Problems in Topology, North-Holland, Amsterdam, 1990, 617-629.

[8] S. Mazurkiewicz, On a certain planar set that has precisely two points in common with each straight line, C. R. Acad. Sci. Lettres Varsovie 7 (1914), 382-384 (in Polish).

[9] - Travaux de topologie et ses applications, Państwowe Wydawnictwo NaukoweÉditions Scientifiques de Pologne, Warszawa, 1969.

[10] A. W. Miller, The axiom of choice and two-point sets in the plane, manuscript, http://www.math.wisc.edu/ ${ }^{\sim}$ miller/res, 2008.

Ben Chad

St Edmund Hall

Oxford, OX1 4AR, UK

E-mail: chad@maths.ox.ac.uk

Rolf Suabedissen

Lady Margaret Hall

Oxford, OX2 6QA, UK

E-mail: suabedis@maths.ox.ac.uk
Robin Knight Worcester College Oxford OX1 2HB, UK E-mail: knight@maths.ox.ac.uk

Received 28 July 2008;

in revised form 10 December 2008 